\title{
Effect of In-plane Mirror Dispersion on Vertical Cavities Based on High-Contrast Grating Mirrors
}

Taghizadeh, Alireza; Mørk, Jesper; Chung, II-Sug

Published in:

Proceedings of 2015 Conference on Lasers and Electro-Optics (CLEO)

Link to article, DOI:

10.1364/CLEO_SI.2015.SW1F.4

Publication date:

2015

Document Version

Peer reviewed version

Link back to DTU Orbit

Citation (APA):

Taghizadeh, A., Mørk, J., \& Chung, I-S. (2015). Effect of In-plane Mirror Dispersion on Vertical Cavities Based on High-Contrast Grating Mirrors. In Proceedings of 2015 Conference on Lasers and Electro-Optics (CLEO) [SW1F.4] Optical Society of America. https://doi.org/10.1364/CLEO SI.2015.SW1F.4

\section{General rights}

Copyright and moral rights for the publications made accessible in the public portal are retained by the authors and/or other copyright owners and it is a condition of accessing publications that users recognise and abide by the legal requirements associated with these rights.

- Users may download and print one copy of any publication from the public portal for the purpose of private study or research.

- You may not further distribute the material or use it for any profit-making activity or commercial gain

- You may freely distribute the URL identifying the publication in the public portal 


\title{
Effect of In-plane Mirror Dispersion on Vertical Cavities Based on High-Contrast Grating Mirrors
}

\author{
Alireza Taghizadeh, Jesper Mørk, and Il-Sug Chung* \\ Department of Photonics Engineering, Technical University of Denmark, DK-2800 Kgs. Lyngby, Denmark \\ *ilch@fotonik.dtu.dk
}

\begin{abstract}
We report how the in-plane dispersion of a high-index-contrast grating reflector influences the transverse mode properties such as shorter wavelengths for lower-order transverse modes and different transverse-mode wavelength spacings for modes with the same size.

(C) 2014 Optical Society of America

OCIS codes: (250.7260) Vertical-cavity surface-emitting lasers; (050.2770) Gratings
\end{abstract}

\section{Introduction}

Recently, the high-index-contrast grating (HCG) is an attractive alternative to the distributed Bragg reflector (DBR) for vertical-cavity surface-emitting lasers (VCSELs), thanks to its broadband high reflectivity [1]. A unique property of the HCG is that it allows to modify the dispersion of the vertical cavity modes in the in-plane directions [2]. This distinct dispersion characteristic results in interesting effects which provides great flexibilities to control the transverse mode sizes, wavelengths, and spatial orders separately in $x$ and $y$ directions.

\section{HCG-based Vertical Cavity}

As shown in Fig. 1(a), our test vertical cavity (VC) consists of a DBR, an air cavity, and an HCG. An HCG is a membrane of high-refractive-index dielectric with one- or two-dimensional grating, surrounded by low-refractiveindex media. In reciprocal lattice space, the HCG modes involved in the reflection process locate near the $\Gamma$ point above the light line. Near the $\Gamma$ point these HCG modes can have either positive or negative dispersion curvature that corresponds to a group velocity in the same or opposite direction to a transverse wavevector, respectively. In Fig. 1(b), the blue and red arrows in the HCG denote negative dispersion case while the green denotes positive case.

(a)

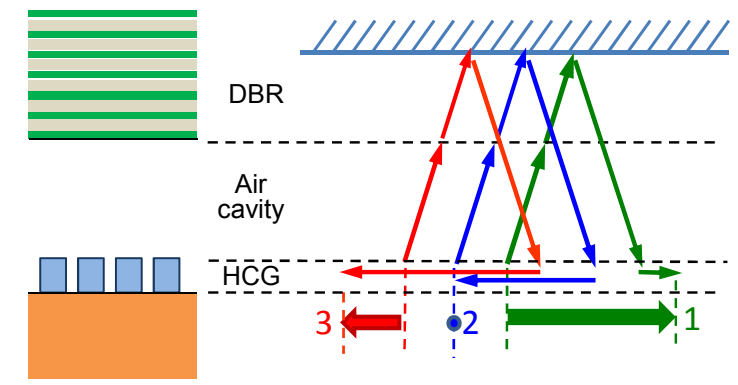

(c)

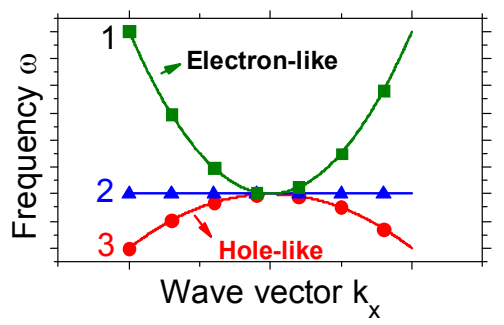

Fig. 1. (a) Schematic of an HCG-based VC. (b) Schematic ray picture of the propagation dispersion of different parts of the HCG-based VC. The blue and red arrows in the HCG denote negative dispersion case while the green denotes positive case. (c) Corresponding cavity dispersions of (b). Case 1 correspond to positive cavity dispersion (electron-like), 2 is for zero cavity dispersion and case 3 is for negative cavity dispersion (hole-like).

In a vertical cavity, the total in-plane dispersion of a cavity mode has contributions from the in-plane dispersions of the two mirrors and the propagation in the nominal cavity. As shown in Fig. 1(b), both the DBR and the propagation dispersion are always positive, while the HCG dispersion can be either positive and negative. Thus, the total cavity dispersion can be positive (case 1), zero (case 2), or negative (case 3). Fig. 1(c) shows these three cases for the test 
vertical cavity designed around $1550 \mathrm{~nm}$ wavelength. By analogy with electron bandstructures, we call the positively and negatively curved cavity dispersion as electron-like and hole-like, respectively. For DBR-based vertical cavities, only the electron-like dispersion can be observed. By changing the HCG dimensions, both the curvature and band edge frequency of the cavity dispersion can be varied.

\section{In-plane Cavity Dispersion Effects}

It is well known that photonic heterostructures can be used to realize longitudinal mode confinement, where the heterostructure is in the direction of light propagation. However, in the case of HCG-based VC photonic hetrostructures are used for the transverse mode confinement [2]. As shown in Fig. 2(a), they can be formed by introducing small variation in grating bar width or period in the well region.

It is well known that higher order transverse modes in VCSELs have higher frequencies or equivalently shorter wavelengths due to their higher spatial $k$ component in the in-plane direction. However in-plane cavity dispersion can change this property. To illustrate the possibility of modifying the fundamental mode property, two HCG-based cavities with positive and negative in-plane dispersions are simulated. For electron-like case (Fig. 2(b)), we have the usual situation of VCSELs; the fundamental mode has the longest wavelength. However, for the hole-like case (Fig. 2(c)), the fundamental mode has shorter wavelength than the higher order modes. As shown in Fig. 2(d), not only the sign but also the magnitude of the mode spacing depends on the band curvature of cavity dispersion. There are several more interesting effects such as bunching of transverse modes in $x$ and $y$ directions, which is observed in our recent experiments [4]. More details will be discussed in the talk.

(a)

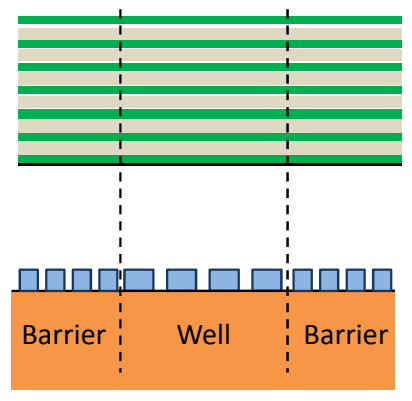

(b)

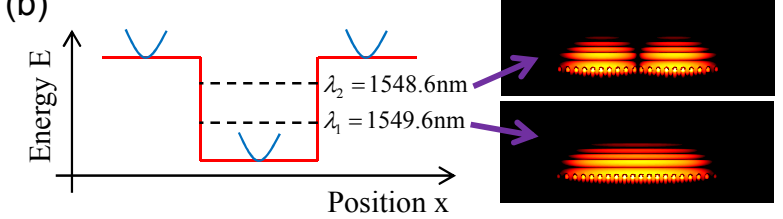

(c)

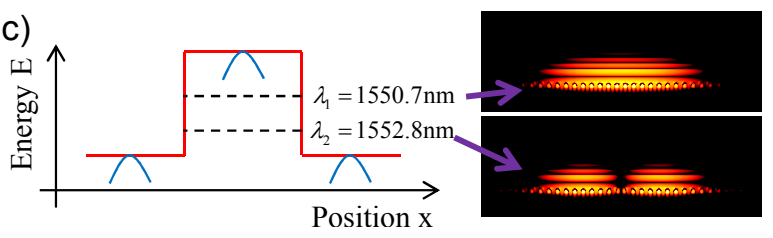

(d)

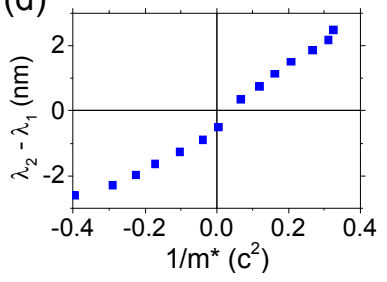

Fig. 2. (a) Schematic of an HCG-based VC with photonic hetrostructure at the boundaries. (b),(c) Transverse spatial variation of photonic bandedge for (b) positive and (c) negative in-plane cavity dispersion respectively. The first two transverse mode profiles are shown with their resonance wavelengths. (d) Transverse-mode wavelength spacing between fundamental and first order mode as a function of cavity dispersion band curvature.

\section{Conclusion}

It has been shown that in-plane dispersion of a high-index-contrast grating reflector can affect several properties of the transverse mode such as shorter wavelengths for lower-order transverse modes and different transverse-mode wavelength spacings for modes with the same size.

\section{References}

1. M. C. Y. Huang, Y. Zhou, and C. J. Chang-Hasnain, "A surface-emitting laser incorporating a high indexcontrast subwavelength grating," Nat. Photonics 1, 119-122 (2007).

2. C. Sciancalepore, et al., "Quasi-3-D light confinement in double photonic crystal reflectors VCSELs for CMOScompatible integration," J. Lightw. Technol. 29(13), 2015-2024 (2011).

3. E. Istrate and E. H. Sargent, "Photonic crystal heterostructures and interfaces," Rev. Mod. Phys. 78(2), 455-481 (2006).

4. G. C. Park, W. Xue, A. Taghizadeh, E. Semenova, K. Yvind, J. Mørk, I.-S. Chung, "Hybrid vertical-cavity laser with lateral emission into a silicon waveguide," arXiv preprint arXiv:1411.2483 (2014). 